\title{
Strategic project selection based on evidential reasoning approach for high-end equipment manufacturing industry
}

\author{
Guangyan Lu, ${ }^{1, *}$, Min Xue ${ }^{2,3}$, Yin $\mathrm{Liu}^{2,3}$, and Wenjun $\mathrm{Chang}^{2,3}$ \\ ${ }^{1}$ Department of Operation Management, CRRC Qishuyan Institute Co., Ltd, Changzhou 213011, Jiangsu, P.R.China \\ ${ }^{2}$ School of Management, Hefei University of Technology, Hefei, Box 270, Hefei 230009, Anhui, P.R. China \\ ${ }^{3}$ Key Laboratory of Process Optimization and Intelligent Decision-making, Ministry of Education, Hefei, Box 270, Hefei 230009, Anhui, \\ P.R. China
}

\begin{abstract}
With the rapid development of science and technology, emerging information technologies have significantly changed the daily life of people. In such context, strategic project selection for high-end equipment manufacturing industries faces more and more complexities and uncertainties with the consideration of several complex criteria. For example, a group of experts rather than a single expert should be invited to select strategic project for high-end equipment manufacturing industries and the experts may feel difficulty to express their preferences towards different strategic projects due to their limited cognitive capabilities. In order to handle these complexities and uncertainties, the criteria framework of strategic project selection is firstly constructed based on the characteristics of high-end equipment manufacturing industries and then evidential reasoning (ER) approach is introduced in this paper to help experts express their uncertain preferences and aggregate these preferences to generate an appropriate strategic project. A real case of strategic project selection in a high-speed train manufacturing enterprise is investigated to demonstrate the validity of the ER approach in solving strategic project selection problem.
\end{abstract}

Keywords. Strategic project selection, high-end equipment manufacturing, evidential reasoning approach, multiple criteria group decision making

\section{Introduction}

High-end equipment manufacturing industries mainly including aviation industry, satellite industry, rail transportation equipment industry, ocean engineering equipment and intelligent manufacturing equipment industry, are considered as an important symbol for the level of science and technology in a nation [1-2]. Emerging information technologies which have driven a new round of revolution in almost all aspects of high-end equipment manufacturing industries, not only create a large amount of opportunities for the development of high-end equipment manufacturing but also bring severe challenges to them. On the one hand, the development of high-end equipment manufacturing enterprises has gradually stepped into a stable stage after experienced a rapid growing stage. On the other hand, the profits and space of the existing market have been constantly compressed and meanwhile the marker shares tend to stabilization.

In order to copy with such challenges to achieve sustainable profits and development, a good choice for high-end equipment manufacturing enterprises is to exploit a new strategic industry or business by selecting an appropriate strategic project [3]. An appropriate strategic project will help high-end equipment manufacturing enterprises expand market shares, increase market profits, improve market reputation to achieve long term and stable competitiveness. However, the problem of selecting a strategic project is not easy in practice [4-5]. Many complex factors should be considered in the process of solving this problem such as the ability to conform the policies of the nation and the industry, market potential, market profitability, the maturity of design and manufacturing technology, human resources and capital resource (See Section 2). Under such condition, it is difficult for a decision maker alone to directly select an appropriate strategic project and a group of experts rather than a single expert alone should be invited. Meanwhile, due to the limited knowledge, experience and cognitive capability of the experts, they may feel uncertain to provide exact preferences about strategic projects. From the above analysis, the strategic project selection can be modeled as a multiple criteria group decision making (MCGDM) problem with uncertainties [6]. An appropriate method is needed to copy with this problem.

With the aim of handling this problem, in this paper, group evidential reasoning (ER) approach, as a useful uncertain MCGDM method, is introduced to help the decision makers select an appropriate strategic project. ER approach [7] is developed based on the decision theory and Dempster-Shafer theory of evidence [8] and allows the decision makers provide their assessments in 
terms of their limited knowledge, experience and cognitive capability by using a distributed belief framework. That is, the decision makers can express their preferences about the degree to which a strategic project is poor, good and average simultaneously. More importantly, the kernel of the ER approach is an ER algorithm to combine assessments provided by each decision maker on each attribute with corresponding weights objectively in order to obtain the collective assessment of each strategic project. Utility is introduced in ER approach to portray the incomplete cognition of the decision maker towards to decision problem. In this situation, utility interval will be generated to generate an appropriate strategic project with the help of minimal satisfaction rule. Finally, a real strategic project selection problem of a high-speed train manufacturing enterprise located in Changzhou, a city in Jiangsu Province of China, is solved by the ER approach.

The rest of this paper is organized as follows. Section 2 constructs the criterion framework for selecting strategic project. Section 3 introduces the ER approach. Section 4 demonstrates a real case of the strategic project selection problem. The paper is concluded in Section 5.

\section{Criteria for strategic project selection}

Strategic project selection plays a key role in the longterm development of high-end equipment manufacturing enterprises. More importantly, how to select an appropriate strategic project is considered as a complex MCGDM problem as mentioned in Introduction. With the aim of solving this problem, criteria framework is firstly constructed by reviewing the existing studies and investigating some enterprises, which includes sixteen criteria belonging to the four perspectives such as business, market, technique and resource as shown in Table 1.

Table 1. Criteria framework for strategic project selection

\begin{tabular}{|c|c|}
\hline \multirow{4}{*}{ Perspective } & Criteria \\
\hline \multirow{4}{*}{ Business } & $\begin{array}{c}\text { Ability to conform the policies of the nation } \\
\text { (PN) }\end{array}$ \\
\cline { 2 - 3 } & $\begin{array}{c}\text { Ability to conform the policies of the industry } \\
\text { (PI) }\end{array}$ \\
\cline { 2 - 3 } & $\begin{array}{c}\text { Capability of being in line with the development } \\
\text { strategy of the enterprise (DSE) }\end{array}$ \\
\hline \multirow{3}{*}{ Market } & Potential capacity of market (CM1) \\
\cline { 2 - 3 } & Potential growth rate of market (GRM) \\
\cline { 2 - 3 } & Competition of market (CM2) \\
\cline { 2 - 3 } & Market Profitability (PM) \\
\hline
\end{tabular}

\begin{tabular}{|c|c|}
\hline \multirow{4}{*}{$\begin{array}{l}\text { Technical } \\
\text { maturity }\end{array}$} & Maturity of design technology (DT) \\
\hline & Maturity of material technology (MT1) \\
\hline & $\begin{array}{l}\text { Maturity of experimental testing technology } \\
\text { (ETT) }\end{array}$ \\
\hline & Maturity of manufacturing technology (MT2) \\
\hline \multirow{5}{*}{$\begin{array}{l}\text { Resource } \\
\text { availability }\end{array}$} & Capital resource (FR) \\
\hline & Human resource (HR) \\
\hline & Equipment resource (ER) \\
\hline & Resource from supply chain (SCR) \\
\hline & Outside Technical support (TS) \\
\hline
\end{tabular}

Table 1 shows that sixteen criteria are included in the framework for the strategic project selection. Several criteria belonging to different perspectives are selected as typical examples to explain their meanings. PN and DSE belonging to the perspective of business mean that the extent to which a project conforms nation policy and development strategy of the enterprise, respectively. PDM and PM belonging to the perspective of market mean the potential size of a market for products and the maximum possible return on an investment, respectively. DT belonging to the perspective of technique means how advanced the proposed design technology is compared with existing design technology. FR and HR belonging to the perspective of resource mean that how much capital and manpower can be obtained, respectively.

\section{Strategic project selection based on ER approach}

In order to copy with strategic project selection, ER approach as a useful MCGDM method is introduced in this section.

\subsection{Modeling of strategic project selection problem}

Suppose that a strategic project selection problem includes $T$ experts $t_{j}(j=1, \ldots, T)$. The relative weights of the $T$ experts on criterion $e_{i}$ for project $a_{l}$ are denoted by $\lambda\left(e_{i}\right)=\left(\lambda^{1}\left(e_{i}\right), \lambda^{2}\left(e_{i}\right), \ldots, \lambda^{T}\left(e_{i}\right)\right)$ such that

$$
0 \leq \lambda^{j}\left(e_{i}\right) \leq 1 \text { and } \sum_{j=1}^{T} \lambda^{j}\left(e_{i}\right)=1 .
$$

All experts are invited to handle a common strategic project selection problem which has $M$ projects $a_{l}(l=$ $1, \ldots, M)$ and $L$ criteria $e_{i}(i=1, \ldots, L)$. The relative weights of the $L$ criteria are denoted by $w=\left(w_{1}, w_{2}, \ldots\right.$, $w_{L}$ ) such that 


$$
0 \leq w_{i} \leq 1 \text { and } \sum_{i=1}^{L} w_{i}=1 .
$$

In general, it may be difficult for the experts to provide completely exact assessments of strategic projects due to their limited knowledge, experience, and cognitive capability. Under such condition, belief distribution in ER approach is a useful tool to help express uncertain assessments.

In ER approach, assume $\Omega=\left\{H_{1}, H_{2}, \ldots, H_{N}\right\}$ symbolizes a set of grades which is increasingly ordered from worst to best. That is, the utilities of grades $u\left(H_{n}\right)$ $(n=1, \ldots, N)$ satisfy the constraint $0=u\left(H_{1}\right)<u\left(H_{2}\right)$ $<\ldots<u\left(H_{N}\right)=1$. The $M$ projects are assessed at the $L$ criteria using $H_{n}(n=1, \ldots, N)$. Let expert $t_{j}$ evaluate project $a_{l}$ on criterion $e_{i}$ to grade $H_{n}$ with a belief degree of $\beta_{n, i}^{j}\left(a_{l}\right)$, then the assessment can be profiled by $B^{j}\left(e_{i}\left(a_{l}\right)\right)=\left\{\left(H_{n}, \beta_{n, i}^{j}\left(a_{l}\right)\right), n=1, \ldots, N ;\left(\Omega, \beta_{\Omega, i}^{j}\left(a_{l}\right)\right)\right\}$, where $\beta_{n, i}^{j}\left(a_{l}\right) \geq 0, \sum_{n=1}^{N} \beta_{n, i}^{j}\left(a_{l}\right) \leq 1$, and $\beta_{\Omega, i}^{j}\left(a_{l}\right)=1$ - $\sum_{n=1}^{N} \beta_{n, i}^{j}\left(a_{l}\right)$ represents the degree of global ignorance. If $\beta_{\Omega, i}^{j}\left(a_{l}\right)=0$, the assessment is complete; otherwise, it is incomplete.

After all projects are assessed by each expert on $L$ criteria, the belief decision matrices will be generated as

$$
D^{j}=B^{j}\left(e_{i}\left(a_{l}\right)\right)_{L \times M} .
$$

\subsection{Aggregation of assessments based on ER approach}

When decision matrices are obtained, the next step is to aggregate assessments of each project provided by $T$ experts on $L$ criteria to generate the collective assessment of each project. In other words, the generation of the collective assessment of project $a_{l}$ includes two steps:

(1) $B^{j}\left(e_{i}\left(a_{l}\right)\right)$ is combined using the ER approach with $\lambda^{j}\left(e_{i}\right)$ to generate the aggregated group assessment of project $a_{l}$ on criterion $e_{i}$ denoted by $B\left(e_{i}\left(a_{l}\right)\right)=\left\{\left(H_{n}\right.\right.$, $\left.\left.\beta_{n, i}\left(a_{l}\right)\right), n=1, \ldots, N ;\left(\Omega, \beta_{\Omega, i}\left(a_{l}\right)\right)\right\}$.

(2) $B\left(e_{i}\left(a_{l}\right)\right)$ is combined using the ER approach again with $w_{i}$ to generate the collective assessment of project $a_{l}$ denoted by $B\left(a_{l}\right)=\left\{\left(H_{n}, \beta_{n}\left(a_{l}\right)\right), n=1, \ldots, N ;(\Omega\right.$, $\left.\left.\beta_{\Omega}\left(a_{l}\right)\right)\right\}$.

In order to complete above aggregation, the ER approach is demonstrated as follows.

Given $D^{j}=B^{j}\left(e_{i}\left(a_{l}\right)\right)_{L \times M}$ where $B^{j}\left(e_{i}\left(a_{l}\right)\right)=\left\{\left(H_{n}\right.\right.$, $\left.\left.\beta_{n, i}^{j}\left(a_{l}\right)\right), n=1, \ldots, N ;\left(\Omega, \beta_{\Omega, i}^{j}\left(a_{l}\right)\right)\right\}$ as modeled in Section 3.1, some associated concepts can be firstly defined for project $a_{l}$ on criteria $e_{i}$.

$$
\begin{aligned}
& m_{n, j}=\lambda^{j}\left(e_{i}\right) \cdot \beta_{n, i}^{j}\left(a_{l}\right), n=1, \ldots, N, j=1, \ldots, T, \\
& m_{\Omega, j}=1-\sum_{n=1}^{N} m_{n, j}=1-\lambda^{j}\left(e_{i}\right) \cdot \sum_{n=1}^{N} \beta_{n, i}^{j}\left(a_{l}\right), j=1, \ldots,
\end{aligned}
$$

$T$,

$$
\vec{m}_{\Omega, j}=1-\lambda^{j}\left(e_{i}\right), j=1, \ldots, T,
$$

$$
\begin{aligned}
& \hat{m}_{\Omega, j}=\lambda^{j}\left(e_{i}\right) \cdot\left(1-\sum_{n=1}^{N} \beta_{n, i}^{j}\left(a_{l}\right)\right), j=1, \ldots, T, \\
& \text { with } m_{\Omega, j}=\vec{m}_{\Omega, j}+\hat{m}_{\Omega, j},
\end{aligned}
$$

where, $m_{n, j}$ means a basic probability mass profiling the degree to which the $j^{\text {th }}$ expert supports the hypothesis that project $a_{l}$ on criteria $e_{j}$ is assessed to the $n^{\text {th }}$ grade $H_{n}$ and $m_{\Omega, j}$ denotes the remaining probability mass unassigned to any individual grade after all $N$ probability masses $m_{n, j}(n=1, \ldots, N)$ have been assessed.

Based on Eqs.(4)-(8), $B\left(e_{i}\left(a_{l}\right)\right)$ can be generated by using the ER approach [7,9] as follows. Given any two assessments denoted by $B^{1}\left(e_{i}\left(a_{l}\right)\right)$ and $B^{2}\left(e_{i}\left(a_{l}\right)\right)$, their aggregated assessment can be represented by $B(2)=$ $\left\{\left(H_{n}, \beta_{n, B(2)}\right), n=1, \ldots, N ;\left(\Omega, \beta_{\Omega, B(2)}\right)\right\}$ with

$$
\begin{gathered}
\beta_{n, B(2)}=\left\{\begin{array}{c}
0 \quad n=\varnothing \\
\frac{\hat{m}_{n, B(2)}}{\sum_{D \subseteq \Omega} \hat{m}_{D, B(2)}} n \subseteq \Omega, n \neq \varnothing
\end{array}\right. \\
\hat{m}_{n, B(2)}=\left[\left(1-w_{2}\right) \cdot m_{n, 1}+\left(1-w_{1}\right) \cdot m_{n, 2}\right]+ \\
\sum_{E \cap F=n} m_{E, 1} \cdot m_{F, 1}, \forall n \subseteq \Omega
\end{gathered}
$$

The residual support of $B(2)$ symbolized by $\hat{m}_{P(\Omega), B(2)}=\left(1-w_{2}\right) \cdot\left(1-w_{1}\right)$ is reassigned back to all elements of $\Omega$ and thus not involved in $B(2)$, as presented in Eq. (9). When the first $j$ assessments are combined, the recursive ER approach is described as follows.

$$
\begin{gathered}
\beta_{n, B(j)}=\left\{\begin{array}{c}
0 \quad n=\varnothing \\
\frac{\hat{m}_{n, B(j)}}{\sum_{D \subseteq \Omega} \hat{m}_{D, B(j)}} n \subseteq \Omega, n \neq \varnothing
\end{array}\right. \\
m_{n, B(j)}=\left\{\begin{array}{c}
0 \quad n=\varnothing \\
\frac{\hat{m}_{n, B(j)}}{\sum_{D \subseteq \Omega} \hat{m}_{D, B(j)}+\hat{m}_{P(\Omega), B(j)}} n \subseteq \Omega, n \neq \varnothing
\end{array},(12)\right. \\
m_{P(\Omega), B(j)}=\frac{\hat{m}_{P(\Omega), B(j)}}{\sum_{D \subseteq \Omega} \hat{m}_{D, B(j)}+\hat{m}_{P(\Omega), B(j)}} \\
\hat{m}_{n, B(j)}=\left[\left(1-w_{i}\right) \cdot m_{n, B(j-1)}+m_{P(\Omega), B(j-1)} \cdot m_{n, j}\right]+ \\
\sum_{E \cap F=n} m_{E, B(j-1)} \cdot m_{F, j}, \forall n \subseteq \Omega, \text { and } \\
\hat{m}_{P(\Omega), B(j)}=\left(1-w_{i}\right) \cdot m_{P(\Omega) B(j-1)},
\end{gathered}
$$

where $0 \leq p_{n, B(j)}, m_{n, B(j)} \leq 1, \forall n \subseteq \Omega, 0 \leq m_{P(\Omega), B(j)} \leq$ 1 , and $\sum_{n \subseteq \Omega} m_{n, B(j)}+m_{P(\Omega), B(j)}=1$ for $j=2, \ldots, T$ recursively.

By using Eqs. (4)-(15), $B\left(e_{i}\left(a_{l}\right)\right)$ can be calculated. After that, $B\left(a_{l}\right)=\left\{\left(H_{n}, \beta_{n}\left(a_{l}\right)\right), n=1, \ldots, N ;\left(\Omega, \beta_{\Omega}\left(a_{l}\right)\right)\right\}$ will be generated by using the recursive ER approach in Eqs. (4)-(15) again. 


\subsection{Generation of an appropriate strategic project}

With the aim of generating an appropriate strategic project, the minimum and maximum expected utilities of project $a_{l}$ (Chin and $\mathrm{Fu} 2014$ ) are calculated as follows.

$$
\begin{gathered}
u_{\min }\left(a_{l}\right)= \\
\sum_{n=2}^{N} \beta_{n}\left(a_{l}\right) u\left(H_{n}\right)+\left(\beta_{1}\left(a_{l}\right)+\beta_{\Omega}\left(a_{l}\right)\right) u\left(H_{1}\right) \\
\quad u_{\max }\left(a_{l}\right)= \\
\sum_{n=1}^{N-1} \beta_{n}\left(a_{l}\right) u\left(H_{n}\right)+\left(\beta_{N}\left(a_{l}\right)+\beta_{\Omega}\left(a_{l}\right)\right) u\left(H_{N}\right)
\end{gathered}
$$

With the aid of $u_{\min }\left(a_{l}\right)$ and $u_{\max }\left(a_{l}\right)$, the minimal satisfaction of project $a_{l}$ can be obtained as

$$
V\left(a_{l}\right)=u_{\text {min }}\left(a_{l}\right)-\max _{m \neq l}\left\{u_{\max }\left(a_{m}\right)\right\}(l=1, \ldots, M)
$$

where $V\left(a_{l}\right) \in[-1,1]$ due to $0 \leq u_{\min }\left(a_{l}\right) \leq u_{\max }\left(a_{l}\right) \leq 1(l=$ $1, \ldots, M) . V\left(a_{l}\right)$ is used to measure the gain from selecting the project $a_{l}$ under the worst case scenario when there is unknown in the performances of any projects. A project with larger minimal satisfaction is more preferred to other project.

\section{Case study}

In this section, a real strategic project selection problem is investigated by the above ER approach.

\subsection{Description of the strategic project selection problem}

In 2016, "the Thirteenth Five-Year Plan for National Economic and Social Development of the People's Republic of China" is enacted to promote the rapid development of the economy and society and improve the level of living in China. The plan puts forward some new requirements for the development of manufacturing industries where an important one is to exploit new industries for the purpose of helping manufacturing industries achieve sustainable competitiveness. Under such condition, a high-end equipment manufacturing enterprise in Changzhou, a city in Jiangsu Province of China, aims to select a strategic project to develop a strategic emerging industry, which is crucial to the longterm development of the enterprise. Following the goal, the enterprise has analyzed its industrial foundation and advantage and further identified four projects as the candidates of strategic projects. They are the driving system of new energy automobile (DSNEA), the RV reducer of industrial robot (RVRIR), the reducer of shield-driven machine (RSDM), and the cleaning machine with high pressure water jet (CMHPWJ).

In order to solve this selection problem, five experts are firstly invited to be the decision makers from three departments which are the department of operation and management, the department of integrated technology and equipment and the department of technology planning and development. Suppose that the five decision makers are denoted by $t_{j}(j=1, \ldots, 5)$, the four projects are denoted by $a_{l}(l=1, \ldots, 4)$, and the sixteen criteria are denoted by $e_{i}(i=1, \ldots, 16)$. After studying the documents concerning the criteria, the decision makers specify the criterion weights denoted by $\mathbf{w}=(0.1$, $0.03,0.045,0.1,0.08,0.08,0.06,0.06,0.05,0.04,0.05$, $0.0625,0.0625,0.03,0.1,0.05)$. The four projects are assessed by each decision maker on all criteria listed in Table 1 by using a set of assessment grades $\Omega=\left\{H_{n}, n=\right.$ $1, \ldots, 5\}=\{$ Very Poor, Poor, Average, Good, Very Good $\}$ $=\{V P, P, A, G, V G\}$. Due to the limited length of this paper, assessments of $a_{l}(l=1, \ldots, 4)$ on $e_{1}$ and $e_{2}$ provided by $t_{j}(j=1, \ldots, 5)$ are demonstrated as an example shown in Table 2. Other assessments are omitted.

Table 2. Assessments on $a_{l}(l=1, \ldots, 4)$ on $e_{i}(i=1,2)$ provided by $t_{j}(j=1, \ldots, 5)$

\begin{tabular}{|c|c|c|}
\hline Criterion & $\boldsymbol{a}_{\mathbf{1}}$ & $\boldsymbol{a}_{\mathbf{2}}$ \\
\hline \multirow{6}{*}{$e_{1}$} & $t_{1}:\{V G, 0.8 ; G, 0.2\}$ & $t_{1}:\{V G, 0.9 ; G, 0.1\}$ \\
& $t_{2}:\{V G, 0.9 ; A, 0.1\}$ & $t_{2}:\{V G, 0.8 ; A, 0.2\}$ \\
& $t_{3}:\{V G, 1\}$ & $t_{3}:\{V G, 1\}$ \\
& $t_{4}:\{V G, 0.9 ; A, 0.05 ;$ & $t_{4}:\{V G, 1\}$ \\
& $P, 0.05\}$ & $t_{5}:\{V G, 1\}$ \\
\hline \multirow{6}{*}{$e_{2}$} & $t_{5}:\{V G, 1\}$ & \\
& $t_{1}:\{V G, 0.9 ; G, 0.1\}$ & $t_{1}:\{V G, 0.9 ; G, 0.1\}$ \\
& $t_{2}:\{V G, 0.8 ; A, 0.2\}$ & $t_{2}:\{V G, 0.8 ; A, 0.2\}$ \\
& $t_{3}:\{V G, 0.2 ; G, 0.8\}$ & $t_{3}:\{V G, 0.2 ; G, 0.8\}$ \\
& $t_{4}:\{V G, 0.8 ; G, 0.1 ;$ & $t_{4}:\{V G, 0.95 ; A, 0.05\}$ \\
& $t_{5}:\{G, 0.8 ; 0.1\}$ & $t_{5}:\{V G, 1\}$ \\
\hline
\end{tabular}

Table 2. (continued) Assessments on $a_{l}(l=1, \ldots, 4)$ on $e_{i}(i=1$, 2) provided by $t_{j}(j=1, \ldots, 5)$

\begin{tabular}{|c|c|c|}
\hline Criterion & $\boldsymbol{a}_{3}$ & $\boldsymbol{a}_{4}$ \\
\hline \multirow{6}{*}{$e_{1}$} & $t_{1}:\{G, 0.6 ; A, 0.3 ;$ & $t_{1}:\{G, 0.5 ; A, 0.5\}$ \\
& $t_{2}:\{G, 0.1\}$ & $t_{2}:\{V G, 0.8 ; A, 0.2\}$ \\
& $t_{3}:\{V G, 0.5 ; G, 0.5\}$ & $t_{3}:\{V G, 0.6 ; G, 0.4\}$ \\
& $t_{4}:\{G, 0.5 ; A, 0.5\}$ & $t_{4}:\{V G, 0.9 ; G, 0.1\}$ \\
& $t_{5}:\{V G, 0.8 ; G, 0.2\}$ & $t_{5}:\{A, 0.9 ; \Omega, 0.1\}$ \\
\hline \multirow{6}{*}{$e_{2}$} & $t_{1}:\{G, 0.6 ; A, 0.4\}$ & $t_{1}:\{A, 0.3 ; P, 0.2 ; \Omega$, \\
& $t_{2}:\{G, 0.6 ; A, 0.4\}$ & $0.3\}$ \\
& $t_{3}:\{V G, 0.3 ; G, 0.7\}$ & $t_{2}:\{V G, 0.7 ; A, 0.3\}$ \\
& $t_{4}:\{G, 0.5 ; A, 0.5\}$ & $t_{3}:\{V G, 0.3 ; G, 0.7\}$ \\
& $t_{5}:\{G, 0.7 ; \Omega, 0.3\}$ & $t_{4}:\{V G, 0.9 ; G, 0.1\}$ \\
& & $t_{5}:\{A, 0.9 ; \Omega, 0.1\}$ \\
\hline
\end{tabular}

\subsection{Generation of a solution to strategic projects selection problem}

To find the solution to the project selection problem, assessments provided by each decision maker on all criteria are aggregated to form collective assessment $B\left(a_{l}\right)$ $(l=1, \ldots, 4)$ by using Eqs. (4)-(15) in Table 3.

Table 3. Collective assessments of the four projects

\begin{tabular}{|l|c|}
\hline$l$ & $B\left(a_{l}\right)(l=1, \ldots, 4)$ \\
\hline
\end{tabular}




\begin{tabular}{|c|c|}
\hline \multirow{2}{*}{1} & $\left\{(V P, 0.0198),\left(P_{2}, 0.052\right),\left(A_{3}, 0.2873\right),\left(G_{4}\right.\right.$, \\
& $\left.0.3226),\left(V G_{5}, 0.299\right),(\Omega, 0.0194)\right\}$ \\
\hline \multirow{2}{*}{2} & $\left\{(V P, 0.0261),\left(P_{2}, 0.1136\right),\left(A_{3}, 0.1767\right),\left(G_{4}\right.\right.$, \\
& $\left.0.2044),\left(V G_{5}, 0.4494\right),(\Omega, 0.0298)\right\}$ \\
\hline \multirow{2}{*}{3} & $\left\{(V P, 0.0098),\left(P_{2}, 0.1196\right),\left(A_{3}, 0.3315\right),\left(G_{4}\right.\right.$, \\
& $\left.0.3365),\left(V G_{5}, 0.1676\right),(\Omega, 0.035)\right\}$ \\
\hline \multirow{2}{*}{4} & $\left\{(V P, 0.0069),\left(P_{2}, 0.0672\right),\left(A_{3}, 0.2485\right),\left(G_{4}\right.\right.$, \\
& $\left.0.3197),\left(V G_{5}, 0.3014\right),(\Omega, 0.0565)\right\}$ \\
\hline
\end{tabular}

The utilities of the grades are provided by the decision maker as follows: $u(V P)=0, u(P)=0.25, u(A)$ $=0.5, u(G)=0.75, u(V G)=1$. By using Eqs. $(16)-(18)$, the minimal satisfaction of each project are calculated in Table 4.

Table 4. The rank-order of the four projects in the strategic project selection problem

\begin{tabular}{|c|c|c|}
\hline Projects & Minimal satisfaction & Ranking \\
\hline DSNEA & -0.0516 & 2 \\
\hline RVRIR & -0.0192 & 1 \\
\hline RSDM & -0.1336 & 4 \\
\hline CMHPWJ & -0.0671 & 3 \\
\hline
\end{tabular}

The ranking order is generated as RVRIR $\succ$ DSNEA $\succ \mathrm{CMHPWJ} \succ \mathrm{RSDM}$ where RVRIR is the most appropriate strategic project in this enterprise. That is, RVRIR is the most appropriate strategic project for this high-speed train manufacturing enterprise.

\subsection{Sensitivity analysis}

In the above problem, the weights of criteria are determined by the group of all experts. Different weights may influence the final values of minimal satisfaction or even final result of the strategic project selection. Thus, two controversial criteria will be further analysed which are capability of being in line with the development strategy of the enterprise $\left(w_{3}\right)$ and market profitability $\left(w_{7}\right)$.

Here, $w_{3}$ is changed from 0.045 to 0.32 with a step of 0.055 . With the variation of $w_{3}$, minimal satisfaction of four projects derived from Eqs. (16) - (18) are changed as plotted in Figure 1. Although the ranking order in Figure 1 is not changed, the values of minimal satisfaction of four projects are significantly changed.

Then, $w_{7}$ is changed from 0.06 to 0.26 with a step of 0.04 . With the variation of $w_{7}$, minimal satisfaction of four projects also derived from Eqs. (16) - (18) are changed as plotted in Figure 2. Figure 2 shows that $a_{4}$ replaces $a_{2}$ to be the most appropriate strategic project when the value of $w_{7}$ increases to about 0.16 . That means the minimal satisfaction is sensitive to the variation of $w_{7}$. In other words, the different importance of market profitability will influence the final solution to the problem of selecting strategic projects.

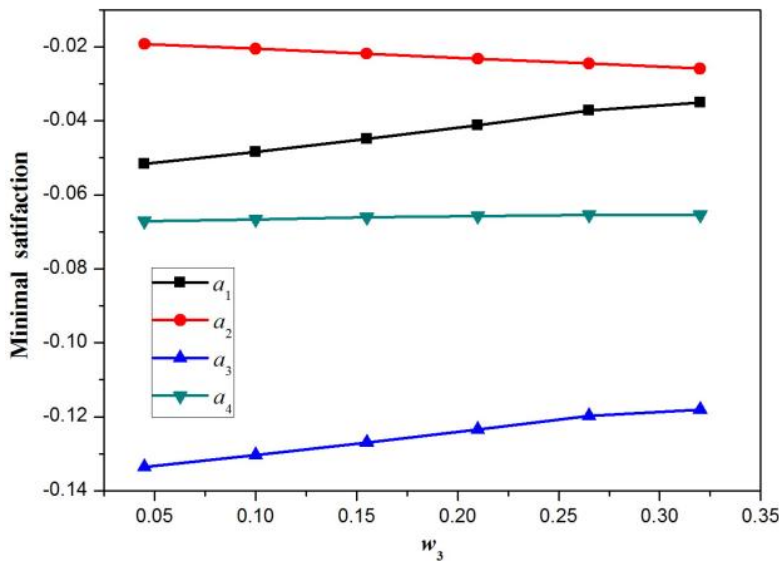

Figure 1. Movement of the minimal satisfaction of the four strategic projects with variation in the value of $w_{3}$

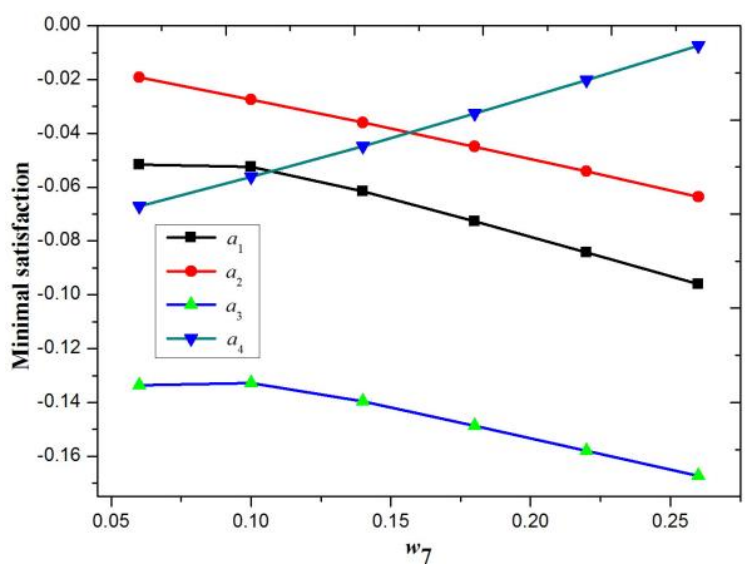

Figure 2. Movement of the minimal satisfaction of the four strategic projects with variation in the value of $w_{7}$

From the above analyses, weights of criteria are very important for selecting an appropriate strategic project. In particular, when the experts consider the weight of market profitability $\left(w_{7}\right)$ should be more than 0.16 , RSDM $\left(a_{4}\right)$ will be the the most appropriate strategic project. The determination of weight of market profitability $\left(w_{7}\right)$ should be more careful.

\section{Conclusion}

In this paper, the strategic project selection problem of high-end equipment manufacturing enterprises is analyzed and investigated based on ER approach. The contributions of this paper are summarized as follows. (1) The criteria framework of selecting strategic project is constructed by analyzing the existing studies. (2) Belief distribution is introduced to help the decision makers express their preferences in strategic project selection flexibility. (3) ER algorithm is demonstrated to combine assessments with weights to generate the best project with the help of utility theory. (4) A real problem of selecting strategic project in a high-speed train manufacturing enterprise is solved by using the ER approach.

In the future, the selected strategic project will be continued analyzed to realize the detailed implement of the project. Moreover, the problem of strategic project 
selection in more high-end equipment manufacturing enterprises will be investigated to further verify the validity of the ER approach. In addition, more complex factors will be considered in the future work of strategic project selection for high-end equipment manufacturing enterprises such as the group consensus requirements and reliability of the experts.

\section{Acknowledgements}

This research is supported by the National Natural Science Foundation of China (Grant Nos. 71571060).

\section{References}

1. T.M. Froese, The impact of emerging information technology on project management for construction, Automation in Construction, 19(5), 531-538, (2010).

2. K. Li, C.S. Shi, Research on classification of suppliers of high-end equipment manufacturing enterprises based on the degree of interdependence. 2013 International Conference on Management Science \& Engineering, 17-19, (2013).

3. K. Khalili-Damaghani, S. Sadi-Nezhad, M. Tavana, Solving multi-period project selection problems with fuzzy goal programming based on TOPSIS and a fuzzy preference relation, Information Sciences, 252, 42-61, (2013).

4. D.J.F Feng, K.H. Huang, Strategic project portfolio selection for national research institutes, Journal of Business Research, 68, 2305-2311, (2015).

5. M.N.M Arratia, I.F. Lopez, S.E. Schaeffer, L. CruzReyes, Static R\&D project portfolio selection in public organizations, Decision Support Systems, 84, 53-63, (2016).

6. M. Tavana, K. Khalili-Damaghani, S. Sadi-Nezhad, A fuzzy group data envelopment analysis model for high-technology project selection: A case study in NASA, Computers \& Industrial Engineering, 66, 10-23, (2013).

7. Yang $\mathrm{J} \mathrm{B}, \mathrm{Xu} \mathrm{D} \mathrm{L}$. On the evidential reasoning algorithm for multiple attribute decision analysis under uncertainty, IEEE Transactions on Systems, Man and Cybernetics-Part A: Systems and Humans, 32(3), 289-304, (2002).

8. Fu C, Yang J B, Yang S L. A group evidential reasoning approach based on expert reliability. European Journal of Operational Research, 246, 886-893, (2015).

9. Yang $\mathrm{J} \mathrm{B}, \mathrm{Xu} \mathrm{D}$ L. Evidential reasoning rule for evidence combination. Artificial Intelligence, 205, 1-29, (2013). 